\title{
Estudio de Caso: Efecto de un Programa de Ejercicio Físico y Consejería Nutricional sobre Variables Físicas y Psicológicas en una Persona con Fibromialgia
}

Case Study: Effect of a Physical Exercise and Nutritional Counseling Program on Physical and Psychological Variables in a Person with Fibromyalgia

\author{
Adrián Alberto Chaves León ${ }^{1}$, María Morera ${ }^{2}$, Miguel Brenes Espinoza ${ }^{3}$ y \\ Sergio Saldaña Quiel ${ }^{4}$ \\ ${ }^{1}$ Gimnasio y SPA ALL IN ONE, Heredia, Costa Rica \\ ${ }^{2}$ Escuela de Ciencias del Movimiento Humano y Calidad de Vida, Universidad \\ Nacional, Costa Rica \\ ${ }^{3}$ Programa de Promoción de la Salud, Área de Salud de Curridabat, Ministerio \\ de Salud, Costa Rica \\ ${ }^{4}$ Consultor Independiente \\ achaves2583@gmail.com
}

\begin{abstract}
Resumen
El propósito de esta investigación fue determinar el efecto de un programa de ejercicio físico, flexibilidad y consejería nutricional sobre las variables físicas (la capacidad aeróbica, la fuerza, la flexibilidad y el perfil lipídico) y las variables psicológicas (el autoconcepto físico, la imagen corporal y la catastrofización del dolor) en una persona con fibromialgia. En este estudio de caso se contó con la participación de una mujer de 53 años de edad. La intervención se aplicó durante ocho semanas, tres veces por semana y con supervisión constante de personal profesional. Antes de la aplicación de la intervención, Lourdes (seudónimo) señala llevar una vida de intensos dolores articulares y musculares que la limitan en el desarrollo de sus actividades cotidianas. Las variables de investigación se midieron al inicio y al final de la intervención. Los resultados demostraron diferencias en el porcentaje de cambio en todas las variables medidas. Además, se evidenció una mejoría en su estado anímico, de acuerdo al testimonio dado por doña Lourdes. Por lo tanto, se concluye que una intervención de ejercicio físico y consejería nutricional es una estrategia efectiva para mejorar la calidad de vida de una persona con fibromialgia.
\end{abstract}

Palabras Claves: Fibromialgia, calidad de vida, catastrofización del dolor, adultez, salud.

\begin{abstract}
The purpose of this research was to determine the effect of an exercise program, flexibility and nutritional counseling on the physical variables (aerobic capacity, strength, flexibility, and the lipid profile) and the psychological variables (physical self-concept, body image and pain catastrophizing) in a person with fibromyalgia. This case study had the participation of 53 years-old woman diagnosed with fibromyalgia (FM). The intervention was implemented for eight weeks, three times a week with the constant supervision of professional staff. Prior to the implementation of the intervention, Lourdes (pseudonym) said to lead a life of severe joint and muscle pain limiting the development of her daily activities. Research variables were measured at the beginning and at the end of the intervention. The results showed differences in the percentage of change in all measured variables. In addition, an improvement in mood was evident, according to the testimony given by Mrs. Lourdes. Therefore, it is concluded that a physical exercise intervention and nutritional counseling is an effective strategy for improving the quality of life of a person with fibromyalgia.
\end{abstract}

Key Words: Fibromyalgia, quality of life, pain catastrophizing, adulthood, health. 


\section{Introducción}

A partir de 1992, la Organización Mundial de la Salud (OMS) reconoce la fibromialgia (FM) como una enfermedad. Esta patología consiste en un síndrome clínico, de etiología desconocida, que provoca dolor musculoesquelético crónico, difuso e incapacitante, el cual en gran medida aleja a quien la padece de su entorno social. Suele acompañarse de trastornos como fatiga, alteraciones del sueño, dolor de cabeza y trastornos gastrointestinales (Arcas, Gálvez, León, Paniagua y Pellicer, 2004), además de la presencia de dolor crónico y generalizado a nivel músculoesquelético y rigidez matutina (García, Rodero, Alda, Sobradiel, Montero y Moreno, 2008). Asimismo, la FM puede presentar parestesias en extremidades, sensación de tumefacción en las manos y cefaleas (Mañex, Fenolloza, Martínez, y Salazar, 2005). Sin embargo, la etiología de esta enfermedad es desconocida, aunque sí afecta el aparato locomotor de las personas que la padecen (García, De Paz, y Márquez, 2012; González, Ortín y Bonillo, 2011).

Como lo afirma Albentosa (2008), la FM engloba diversas patologías y no siempre tiene un mismo origen. Según García et al. (2008), la presencia de dolor localizado en puntos específicos proporciona parte del diagnóstico para la misma. Con frecuencia este dolor se da de manera hipersensible, cuya sensación se manifiesta por simple tacto, situación que no se observa en sujetos sanos (González et al., 2012). Produce además un detrimento del estado de ánimo, lo que afecta la salud psicológica (García et al., 2012). Mañex et al. (2005) describen la FM como una forma de reumatismo no articular, caracterizada por la presencia de dolor en los cuatro cuadrantes del cuerpo por más de tres meses. En estos cuadrantes se identifican 18 puntos anatómicos en los que se percibe dicho dolor y la fibromialgia se diagnostica tras la existencia de 11 puntos de los 18 correspondientes.

Aparicio, Ortega, Heredia, Carbonell y Delgado (2011) destacan que en la fibromialgia las personas perciben una condición física reducida y una fatiga crónica, síntomas que limitan las actividades en su diario vivir, como caminar, levantar o transportar objetos. Sin embargo, el ejercicio físico es considerado uno de los principales métodos en el manejo de las enfermedades crónicas (Pedersen y Saltin, 2006). Se ha encontrado que el ejercicio físico puede tener un efecto positivo sobre aspectos relacionados con la calidad de vida, la percepción del dolor y la capacidad funcional en pacientes con FM. Un estudio llevado a cabo por Estrada, Munguía, Legaz y Serrano (2003), utilizando el SF-36 Health Survey con un $n=79$ dividido en 3 grupos, concluye que un programa de actividad física aplicado a personas con FM, basado en aeróbicos de bajo impacto, contribuye a mejorar la calidad de vida de pacientes con fibromialgia.

En el caso de patologías asintomáticas, algunos estudios como los realizados por Gurer, Faruk y Ay (2006) han vinculado la fibromialgia a los estados generales de perfil lipídico en las personas, condicionados por los valores de triglicéridos, colesterol total (CT), lipoproteínas de baja densidad (LDL) y proteínas de alta densidad (HDL). Un estudio llevado a cabo por Aparicio, Ortega, Heredia, Carbonell y Delgado (2011) destaca que en mujeres con FM, estas presentaban un $28 \mathrm{~kg} / \mathrm{m}^{2}$ y un porcentaje de grasa de $38.6 \%$. Otro ejemplo de esta vinculación es el estudio de Cordero et al. (2012), donde se concluye que un $57.9 \%$ de mujeres con FM poseen niveles de CT alterados, un $63.4 \%$ de aumento en las LDL y un $19.9 \%$ por encima de lo recomendado en los valores de triglicéridos. Por su parte, Arranz, Canela y Rafecas (2012), mediante un estudio en 103 mujeres con FM, mostraron que altos 
valores del IMC en dicha población guardan alta correlación negativa con la salud emocional y mental.

Los beneficios que brinda la actividad física a nivel psicológico pueden promover la salud mental. García et al. (2012) agregan que la disciplina, la actitud y el empeño que impone un plan de ejercicio para su realización, conllevan al desarrollo de la autoeficacia y el desarrollo de la confianza en las personas con FM para sentirse capaces de ejecutar y finalizar dicho plan, y esto a su vez mejora las percepciones de un individuo hacia sí mismo a nivel físico y cognitivo.

Otro constructo de importancia es el autoconcepto. Según Cox (2008), este se refiere a las creencias, ideas, emociones, sentimientos y actitudes que un individuo tiene acerca de sí mismo. Moreno, Garrosa y Gálvez (2005) mencionan que la personalidad de un individuo depende no solo del entorno social, sino también de la representación cognitiva subjetiva de sí mismo, es decir, de la percepción e información que la persona capta de sí misma, fundamentada en las experiencias que ha vivido durante las diferentes etapas del crecimiento. Un estudio realizado por Garaigordobil y Govillard (2014), en el que participaron 140 personas con FM y 50 personas no afectadas con dicha enfermedad, mostró que los individuos con FM tienen un nivel superior de discapacidad funcional y dolor, además de una baja autoestima y autoconcepto. García, De Paz y Márquez (2012), a partir de una investigación de 12 semanas en la que formaron parte 28 mujeres diagnosticas con FM, mostraron que 3 sesiones semanales de ejercicio aeróbico supervisado mejoran significativamente la función física, el funcionamiento social, la autoestima y el autoconcepto, sin encontrar beneficios con respecto al ejercicio isométrico, la resistencia muscular y la flexibilidad.

Vinculada a la actividad física (AC), se encuentra la autoimagen corporal que se define como "la imagen y representación cognitiva que una persona tiene de su propio cuerpo" (Cox 2008, p.417). La forma como una persona interpreta su figura corporal puede no estar apegada a la realidad, lo que hará que otras percepciones tales como la autoestima y el autoconcepto igualmente se vean alteradas en todas sus dimensiones (Castillo y Moncada, 2010). Según afirman Sepúlveda, Botella y León (2001), desde una perspectiva más dinámica, la imagen corporal (IC) se define en términos de sentimientos y actitudes hacia el propio cuerpo. Un estudio realizado por Akkaya, Akkaya, Simsir, Ceyhan y Sahin (2012) en 51 pacientes con FM y 41 sujetos sanos utilizados como grupo control, concluyó que las personas con FM poseen una imagen corporal alterada en comparación con sujetos sanos.

Otra variable a tomar en cuenta en personas con FM es la catastrofización del dolor, la cual se define como una orientación negativa y exagerada de dicho dolor, que se desarrolla a partir de aspectos psicológicos como inseguridad y ansiedad. Además, debido a esto se manifiesta un mayor consumo de medicamentos, pronósticos perturbadores e incluso ideas suicidas (García et al., 2008). Osman, Barrios, Gutiérrez, Kopper, Merrielfd y Grittmann (2000) mencionan que la catastrofización del dolor se asocia a factores cognitivos relacionados con la percepción individual del dolor de forma persistente. García et al. (2008) apuntan que, independientemente del grado de depresión, la catastrofización está relacionada con un incremento de la actividad cerebral en las áreas relacionadas con la anticipación del dolor, los aspectos emocionales y el control motor. Las evidencias señalan que hay efectos positivos a nivel psicosomático al combinar el ejercicio físico y la relajación, utilizando el ejercicio físico no solo como un determinante antropométrico, sino también como un medio Revista MHSalud® (ISSN: 1659-097X) Vol. 11. No. 2. Febrero-Agosto, 2015

Artículo protegido por Licencia Creative Commons Attibution-NonComercial-NoDerivs 3.0 Costa Rica C5) (1) (9) 
de interacción social para incrementar el bienestar subjetivo (Moreno, Garrosa y Gálvez, 2005).

González, Ortín y Bonillo (2011) realizaron una investigación en la que participaron 42 mujeres con fibromialgia, las cuales fueron integradas a tres grupos de los cuales uno realizó actividad física, otro recibió una intervención psicológica y al tercero se le brindó una intervención tanto con ejercicio físico como con atención psicológica. Los resultados del estudio mostraron que el grupo que realizó ejercicio físico conjuntamente con ayuda psicológica tuvo un mejor control de la depresión y la ansiedad, indicadores relacionados, según Bragado, Hernández, Sánchez y Urbano (2008), con una baja autoestima y un bajo autoconcepto. Además, los investigadores concluyen que se debería incluir en el estilo de vida el ejercicio físico como forma de terapia no farmacológica, en el cual se lleven a cabo ejercicios para mejorar la flexibilidad, la fuerza muscular y la capacidad aeróbica. Dichas intervenciones, sumadas a una correcta alimentación, podrían ayudar a mejorar la capacidad funcional de la persona afectada y con esto optimizar su calidad de vida y su estado de salud general. Por tanto, el propósito de esta investigación fue determinar el efecto de un programa de ejercicio físico, flexibilidad y consejería nutricional sobre las variables físicas (la capacidad aeróbica, la fuerza, la flexibilidad, el perfil lipídico) y las variables psicológicas (el autoconcepto físico, la imagen corporal y la catastrofización del dolor) en una persona con fibromialgia.

\section{Metodología}

\section{Caso de estudio.}

Una mujer de 53 años, divorciada, madre de dos hijos, quien trabaja tiempo completo cuidando niños y haciendo labores del hogar. Pertenece a la clase media-baja de la provincia de Heredia, Costa Rica. Ella fue diagnosticada con fibromialgia desde hace 5 años. Presentó previamente a la intervención una talla de $1.59 \mathrm{~cm}$, un porcentaje de grasa 49.5, un peso 95.5 $\mathrm{Kg}$ y un IMC $37.89 \mathrm{Kg} / \mathrm{m}^{2}$. Se realizaron exámenes de laboratorio (hematológicos y glucosa) únicamente para determinar el estado de salud general previo de la paciente. Se identificaron en ella valores normales de hemoglobina, eritrocitos y VCM, descartando por este medio cualquier patología no identificada que pudiera perjudicar la salud de la paciente. Los niveles de perfil lipídico previos a la intervención fueron un colesterol total de $215 \mathrm{mg} / \mathrm{dL}$, el HDL $45 \mathrm{mg} / \mathrm{dL}$, LDL de $140 \mathrm{mg} / \mathrm{dL}$ y los Triglicéridos en $150 \mathrm{mg} / \mathrm{dL}$. En adelante se referirá a ella con el seudónimo de "Lourdes".

\section{Instrumentos.}

\section{Capacidad aeróbica.}

La prueba de caminata de 6 minutos (PM6M) fue utilizada para evaluar de forma submáxima la capacidad aeróbica de la persona. Para realizar esta prueba se requiere de un pasillo de al menos 30 metros cuya superficie sea plana. Se determina la distancia que la persona puede recorrer caminando lo más rápido posible durante 6 minutos. Antes y después de la prueba se deben medir la frecuencia cardiaca y la presión arterial, las cuales junto con los datos personales del paciente específicos para la prueba, se utilizarán para el desarrollo de ecuaciones que permitan determinar el VO2 submáximo (Giraldo, 2008). Para esta prueba también se utilizó el monitor cardiaco y GPS marca "Garmin", que permite determinar la distancia alcanzada en la prueba de 6 minutos y la frecuencia cardiaca correspondiente a cada metro recorrido. Los monitores cardiacos marca Garmin Forerunner 305 tienen un margen de 
error aceptable de hasta $8 \%$ (Garmin Iberia, 2014) y mediante un test-retest se estableció la fiabilidad de la PM6M de un 0.95 (Harada, Chiu y Stewart, 1999).

Como parte del protocolo de la PM6M, también se utilizó un medidor digital de presión arterial marca "Citizen", modelo $\mathrm{CH}-432 \mathrm{~B}$, para medir la presión arterial de la persona en estudio. Este medidor posee los criterios de validación con diferencias medias entre el dispositivo Citizen $\mathrm{CH}-432 \mathrm{~B}$ y un esfigmomanómetro de mercurio es de 0.7 +/- 4.4 $\mathrm{mmHg}$ para la presión sistólica y de $-3.6+/-4.0 \mathrm{mmHg}$ para la presión diastólica, cumpliendo así con los criterios de aprobación de la European Society of Hypertension (Cotte, Faltenbacher, Willich y Bogner, 2008).

\section{Flexibilidad.}

Se empleó la Prueba de Sit and Reach para medir el grado de flexibilidad de la persona en estudio. El protocolo utilizado fue el siguiente: la persona ejecutante debe estar sentada en el suelo con las piernas extendidas, la planta de los pies pegadas al cajón de flexibilidad y apoyando su espalda y su cabeza sobre una pared, sus brazos deberán estar extendidos para delante donde deberá llevar las manos al frente, una superpuesta sobre la otra y la punta de los dedos en contacto con la regla. La persona evaluadora deberá marcar ese punto como el punto cero. Tras esta posición, la persona ejecutante inicia el test deslizando las manos sobre el cajón debiendo lograr alcanzar la máxima distancia con sus manos. De acuerdo con el estudio fiabilidad y validez de las pruebas Sit and Reach, revisión sistemática, la prueba Sit and Reach cuenta con un índice de correlación intraclase (ICC) de 0.89-0.99, lo que indica una elevada fiabilidad, sin embargo presenta una validez moderada para determinar la flexibilidad de los isquiotibiales y lumbares, con valores que rondan los 0.37-0.77 para hombres y 0.370.85 para mujeres (Ayala, Sainz de Baranda, de Ste Croix y Santoja, 2012).

\section{Peso, talla y porcentaje de grasa.}

Se empleó para medir el peso y el porcentaje de grasa de la sujeto en estudio una báscula marca "Tanita", modelo UM-081. Esta permite determinar esos datos mediante bioimpedancia eléctrica. Dicho método posee un margen de error $+/-600 \mathrm{~g}$ para pesos que van de $0 \mathrm{~kg}$ a $75 \mathrm{~kg},+/-800 \mathrm{~g}$ para pesos que van de $75 \mathrm{~kg}$ a $100 \mathrm{~kg}$ y de $+/-1200 \mathrm{~g}$ para pesos que van de $100 \mathrm{~kg}$ a $150 \mathrm{~kg}$ (Duque, 2014).

La talla fue medida por medio de una cinta métrica en centímetros, se coloca a la persona a evaluar con la espalda y talones junto a la pared en la cual se encuentra el tallímetro.

\section{Perfil lipídico.}

Mediante exámenes de laboratorio se determinaron los valores de colesterol total, lipoproteínas de alta de densidad, lipoproteínas de baja densidad y triglicéridos.

\section{Catastrofización del dolor.}

La escala Pain Catastrophizing Scale (siglas en inglés PCS), traducida al español como la Escala de la Catastrofización ante el Dolor, versión española (García et al, 2008), es una escala autoadministrada de 13 ítems cuyo propósito es determinar el nivel el dolor percibido por la persona. Cada ítem se puntúa de 0 (nada) a 4 (siempre) y las puntuaciones totales van de 0 a 52. Comprende 3 dimensiones: a) rumiación; b) magnificación, y c) desesperanza. Posee un alfa de Cronbach de 0.79, además una fiabilidad de 0.84 y sensibilidad al cambio

Revista MHSalud® (ISSN: 1659-097X) Vol. 11. No. 2. Febrero-Agosto, 2015 
(tamaño de efecto $\geq 2$ ). Este instrumento se utilizó para determinar el grado de autointerpretación del dolor con relación a la fibromialgia.

\section{Imagen corporal.}

Se utilizó la escala Contour Drawing Rating Scale (CDR), elaborado por Thompson y Gray (1995), traducida al español como Test de Siluetas para establecer la imagen corporal que la persona en estudio tiene de sí misma y la imagen corporal que considera como ideal. Está compuesta por 9 dibujos que identifican la forma física, desde el valor 1 como muy delgado hasta el valor 9 como muy obesos, tanto de hombres como de mujeres, de las cuales el sujeto debe discrepar entre su figura actual y su figura ideal. A la persona se le solicita que identifique con una "I" la figura que considera su ideal y con una "A" su figura actual. El coeficiente de Pearson de fiabilidad para dicha prueba mediante test-retest es $\mathrm{r}=78$ (Rodríguez y Cruz, 2006).

\section{Autoconcepto físico.}

Se empleó el Cuestionario de Autoconcepto Físico (CAF) para valorar el autoconcepto físico de la sujeto en estudio. Este cuestionario (Goñi, Ruiz y Liberal, 2004) toma como base los modelos del autoconcepto más importantes propuestos por Fox (1997). Dicho cuestionario consta de 36 ítems comprendidos primeramente en cuatro subescalas de autoconcepto físico, los cuales son: atractivo físico, habilidad física, condición física y fuerza, además de dos subescalas generales que constan del autoconcepto físico general y el autoconcepto general. De los 36 ítems, unos están redactados de forma positiva, por ejemplo: "tengo mucha resistencia física", los cuales se puntúan en una escala tipo Likert, de 1 a 5 (desde falso a verdadero respectivamente), y otros están redactados de forma negativa "no me gusta mi imagen corporal", puntuados de 5 a 1 (de falso a verdadero respectivamente). El máximo de puntos a obtener es 180. El puntaje de cada subescala se obtiene mediante la sumatoria de las puntuaciones de todos los ítems pertenecientes a dicha subescala. La confiabilidad de dicho cuestionario está determinada por el alfa de Cronbach $=0.93$. Todas las escalas ofrecen un nivel alto de fiabilidad, cuyos valores son 0.87 para el atractivo físico, 0.84 para la habilidad física, 0.88 para la condición física, 0.83 para la fuerza, 0.86 para el autoconcepto físico general y 0.84 para el autoconcepto general (Goñi, Ruiz y Liberal, 2004).

\section{Procedimiento.}

Primeramente se realizó una búsqueda en un barrio de Heredia de personas que tuvieran algún tipo de padecimiento y en esta búsqueda se dio con doña Lourdes, quien se ofreció a realizar el estudio de caso. Una vez seleccionada la participante de la investigación, se realizó una entrevista para determinar la edad, las características familiares, laborales y del entorno donde ella se desenvuelve. Además, se recolectó información sobre su patología y se le realizó los exámenes de laboratorio (hematológicos, perfil lipídico, glucosa).

Una semana después se efectuaron las mediciones de la variables en estudio, en el siguiente orden: talla, peso, porcentaje de grasa, prueba de Sit and Reach y la prueba PM6M. Antes y después de la prueba de caminata se midió la FC y la presión arterial para la obtención posterior del $\mathrm{VO}^{2}$ submáximo. En otro día de esa semana se le administró los tres cuestionarios psicológicos (Pain Catastrophizing Scale, CAF, Test de Siluetas) y la prueba de flexibilidad. Durante esa misma semana la persona profesional en nutrición la evaluó para determinar el plan nutricional a seguir. 


\section{Intervención.}

El programa de ejercicio físico y consejería nutricional fue elaborado por dos especialistas en el área del Movimiento Humano, un fisioterapeuta y una nutricionista. Este programa fue planificado y adaptado a las condiciones de la paciente, tanto en disposición de horario como en el lugar de ejecución de las actividades (por ejemplo, diferentes espacios en su hogar o al aire libre). Esta intervención se desarrolló durante 8 semanas como parte de un proyecto de estudio preestablecido, el cual se realizó 3 veces por semana, con una duración por sesión entre 45 a 60 minutos. Dos de las sesiones por semana se dedicaron al ejercicio físico con supervisión constante del educador físico y el tercer día se destinó a la terapia de relajación y al ejercicio de flexibilidad, dados por un fisioterapeuta.

El programa de ejercicio físico consistió en trabajo aeróbico y de contrarresistencia. En el caso del trabajo aeróbico, se utilizó la caminata, el subir y bajar gradas, y movimientos articulares continuos como métodos para mejorar la capacidad aeróbica. Para el trabajo de contrarresistencia de las principales zonas musculares del cuerpo (espalda, piernas, pecho, brazos, zona abdominal y lumbar) se empleó ligas, el propio peso del cuerpo y pesas de arena

Cabe recalcar que este programa de intervención también incorporó un diálogo constante de doble vía entre las personas profesionales y doña Lourdes, esto con el fin de conocer el estado motivacional y el bienestar de doña Lourdes semana a semana. Una vez finalizada la intervención, se volvieron a medir todas las variables físicas y psicológicas.

\section{Análisis estadístico.}

Al ser un estudio de caso clínico los resultados obtenidos fueron analizados a nivel cualitativo y se utilizó el porcentaje de cambio como parámetro para comparar los valores iniciales contra los valores finales y a su vez con valores de normalidad. La fórmula utilizada para determinar el porcentaje de cambio fue:

$$
\% \text { cambio }=\frac{\text { post }- \text { pre }}{\text { pre }} * 100
$$

\section{Resultados}

Los resultados de la Tabla 1 muestran los valores obtenidos en el pre-test y el pos-test respecto a la PM6M, la flexibilidad, el porcentaje de grasa y el peso. En el caso de la FC en reposo, se presentó una disminución de 14 latidos por minuto, lo que representa un porcentaje de cambio de -16.27. Además, aumentó en $1.92 \mathrm{ml} / \mathrm{kg} / \mathrm{min}$ el consumo de oxígeno submáximo, representando un porcentaje de cambio de +11.49 , y la PAS disminuyó 26 $\mathrm{mmHg}$, lo que representa un porcentaje de cambio de -15.66 , mientras que la PAD no presentó variaciones. Respecto a la composición corporal, el porcentaje de grasa disminuyó 7.9 para un porcentaje de cambio -13.78. Asimismo, el IMC disminuyó $2.46 \mathrm{Kg} / \mathrm{m}^{2}$, lo que representa un porcentaje de cambio -3.51. Por último, la distancia en la prueba de 6 minutos aumentó 117 metros, para un porcentaje de cambio de +24.33 , y la flexibilidad coxofemoral aumentó $7 \mathrm{~cm}$, para un porcentaje de cambio de +33.3 . 
Tabla 1

Valores pre-test, post-test y porcentaje de cambio de las variables físicas

\begin{tabular}{lcccc}
\hline Variables físicas & Pre-test & Post-test & Valores normales & \% de cambio \\
\hline Distancia prueba 6 min & $483 \mathrm{~m}$ & $600 \mathrm{~m}$ & $<300 \mathrm{~m}$ & +24.22 \\
FC reposo (p/m) & 86 & 72 & 60 & -16.27 \\
$\mathrm{VO}_{2}$ submax (ml/kg/min) & 17 & 18.92 & $>31$ & +11.49 \\
PAS/PAD (mmHg) & $166 / 88$ & $140 / 88$ & $<120 /<80$ & $-15.66 / 0$ \\
Flexibilidad (cm) & 21 & 28 & 30 & +33.3 \\
\%grasa & 57.3 & 49.4 & $25-27$ & -13.78 \\
Peso & 95.5 & 89.6 & 62.74 & -6.17
\end{tabular}

Nota: FC: frecuencia cardiaca, $\mathrm{VO}_{2}$ max: consumo máximo de oxígeno, PAS: presión arterial sistólica, PAD: presión arterial diastólica, IMC: índice de masa grasa. Valores normales según la ACSM (2005).

La figura 1 muestra el comportamiento de la FC durante la realización de la prueba de 6 minutos en el pre-test y el post-test. Se puede observar cómo a pesar de que en el post-test mejoró la distancia recorrida, la cantidad de latidos fue menor a la obtenida en el pre-test, lo que indica un menor esfuerzo cardiovascular para realizar la prueba.

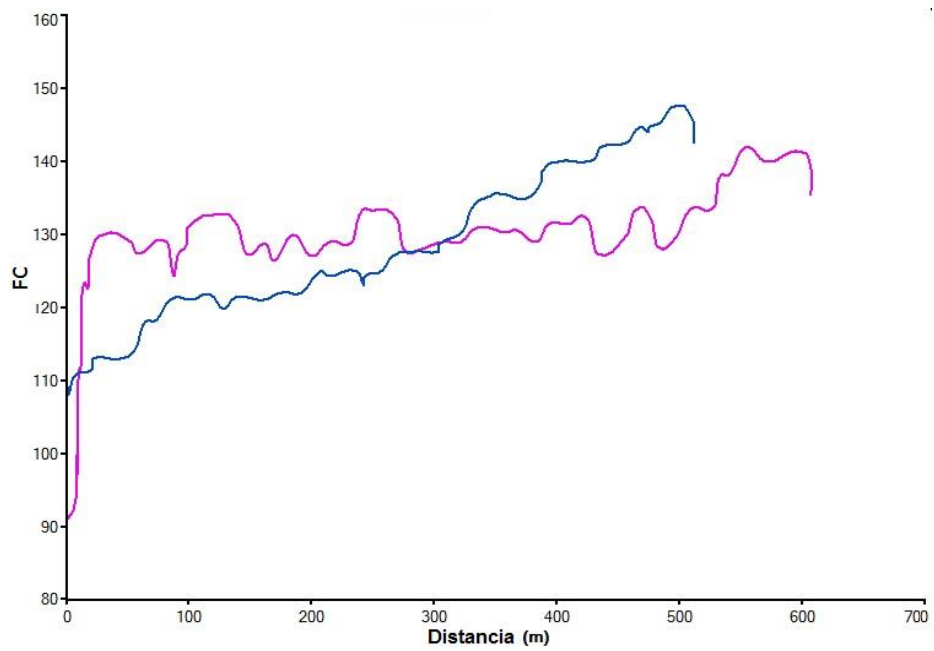

Figura 1. Comportamiento de la FC en el pre-test (color azul) y el post-test (color rosado), según la distancia.

En la tabla 2 se pueden encontrar los resultados entre el pre-test y el pos-test del perfil lipídico, los cuales muestran una disminución en el porcentaje de cambio de CT, LDL y Triglicéridos, y un aumento del porcentaje de HDL (ver Tabla 1).

Tabla 2

Valores pre-test, post-test y porcentaje de cambio del perfil lipídico

\begin{tabular}{lcccc}
\hline Perfil lipídico & $\begin{array}{c}\text { Valores } \\
\text { pre-test }\end{array}$ & $\begin{array}{c}\text { Valores } \\
\text { post-test }\end{array}$ & $\begin{array}{c}\text { Valores } \\
\text { normales }\end{array}$ & $\begin{array}{c}\% \text { de } \\
\text { cambio }\end{array}$ \\
\hline Colesterol total $(\mathrm{mg} / \mathrm{dL})$ & 215 & 180 & $<200$ & -16.27 \\
Lipoproteínas de baja densidad (mg/dL) & 140 & 117 & $<130$ & -16.42 \\
Lipoproteínas de alta densidad(mg/dL) & 45 & 46 & $>40$ & +2.22 \\
Triglicéridos (mg/dL) & 150 & 82 & $<150$ & -45.33 \\
\hline
\end{tabular}

Nota: Los valores normales son los establecidos por NIH (2002) y ACSM (2005).

Revista MHSalud® (ISSN: 1659-097X) Vol. 11. No. 2. Febrero-Agosto, 2015

Artículo protegido por Licencia Creative Commons Attibution-NonComercial-NoDerivs 3.0 Costa Rica 
La tabla 3 muestra una disminución de la catastrofización del dolor que realizó la paciente antes y después de la intervención. En las diferentes dimensiones del PCS, la paciente disminuyó la percepción del dolor y obtuvo un mejor pronóstico del mismo hacia el futuro, lo cual se refleja en el valor total del post-test en comparación al pre-test.

Tabla 3

Valores pre-test, post-test y porcentaje de cambio de las dimensiones del PCS

\begin{tabular}{lccc}
\hline Dimensiones & Pre-test & Post-test & \% de cambio \\
\hline Rumiación & 17 & 4 & -76.47 \\
Magnificación & 12 & 3 & -75 \\
Desesperanza & 15 & 10 & -33.33 \\
\hline Total & 47 & 17 & -63.82 \\
\hline
\end{tabular}

Los resultados del test de autoimagen demuestran una disminución en el valor percibido posterior a la intervención, pasando de la figura número 9 a la 8 . La imagen corporal A (ver figura 2) fue la identificada por la sujeto como la asociada a su percepción pre-test. Por otra parte, la imagen corporal I (ver figura 2) determina la figura corporal ideal según el criterio de la persona evaluada. Respecto a dicha figura y previo a la intervención, la paciente identificó la imagen corporal 4 (ver figura 2) y posterior a la intervención seleccionó la imagen corporal 5, optando por una opción más realista y de aceptación propia según lo manifestado posterior a la realización post-test.

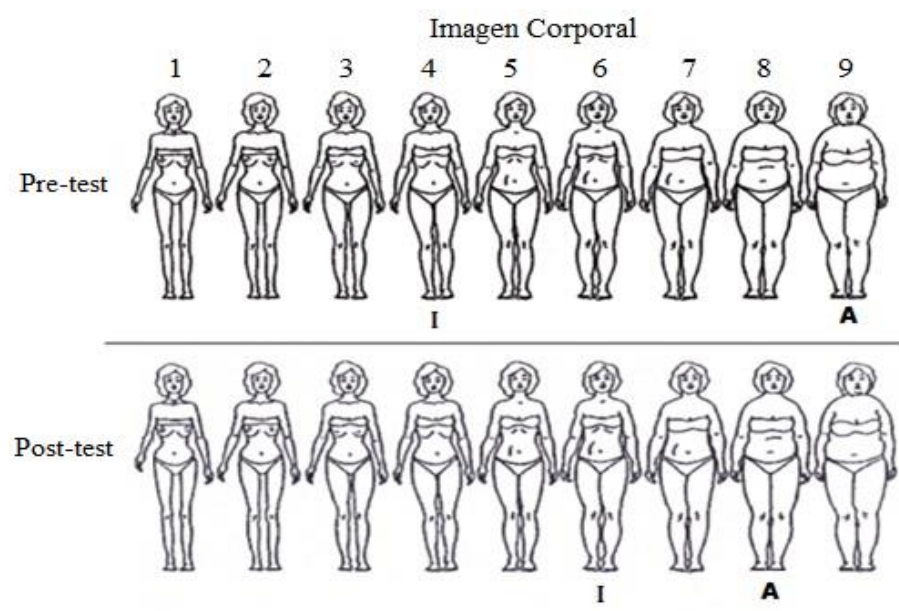

Figura 2. Resultados del test de autoimagen antes y después de la intervención, mediante el instrumento Contour Drawing Rating Scale de Thompson y Gray (1995).

La tabla 4 muestra que todos los valores de autoconcepto físico en el post-test, en sus respectivas dimensiones, aumentaron el valor con respecto al pre-test. En el resultado total al final de la intervención se muestra un aumento de 63 puntos en la percepción del autoconcepto físico total entre el pre-test y el pos-test, lo que constituye un porcentaje de cambio de 71.59. 
Tabla 4

Valores pre-test y post-test obtenidos en las dimensiones del autoconcepto físico

\begin{tabular}{lccc}
\hline Dimensión & Pre-test & $\begin{array}{c}\text { Pos- } \\
\text { test }\end{array}$ & \% de cambio \\
\hline Habilidad física & 9 & 22 & 144.4 \\
Condición física & 14 & 27 & 92.85 \\
Fuerza & 14 & 24 & 71.42 \\
Atractivo físico & 18 & 27 & 50 \\
Autoconcepto físico general & 12 & 28 & 133.33 \\
Autoconcepto general & 21 & 23 & 9.52 \\
\hline Total & 88 & 151 & 71.59 \\
\hline
\end{tabular}

\section{Discusión}

En el caso de Lourdes, todas las variables evaluadas relacionadas con la capacidad funcional mostraron cambios positivos, lo cual se vio reflejado en un mejor desempeño aeróbico en la prueba de 6 minutos, en la cual a pesar de alcanzar una mayor distancia en el post-test, esta se finalizó con una frecuencia cardiaca menor. Los valores de las variables que constituyen dicha prueba fueron los siguientes: la presión arterial se encontraba en 166/88 $\mathrm{mmHg}$ y con una frecuencia cardiaca de $86 \mathrm{p} / \mathrm{m}$, valores que disminuyeron a $140 / 88 \mathrm{mmHg}$ y $72 \mathrm{p} / \mathrm{m}$, respectivamente, posterior a la intervención, y el $\mathrm{VO}_{2}$ aumentó de $17 \mathrm{ml} / \mathrm{kg} / \mathrm{min}$ a $18.92 \mathrm{ml} / \mathrm{kg} / \mathrm{min}$.

Por esta razón, el ejercicio físico representa un importante instrumento para tratar la obesidad, como lo fue en el caso de Lourdes, quien obtuvo una disminución de un $57.3 \%$ de grasa a un $49.4 \%$, así como una disminución del peso de $95.5 \mathrm{Kg}$ a $89.0 \mathrm{Kg}$, que refleja a su vez una disminución del IMC de $37.89 \mathrm{Kg} / \mathrm{m}^{2}$ a $35.32 \mathrm{Kg} / \mathrm{m}^{2}$. Esto representa, de acuerdo con Grundy et al. (2004), una disminución de complicaciones en su salud cardiovascular y una mejora en la calidad de vida.

Para valorar la flexibilidad de la paciente, se aplicó el test de sit and reach, con el cual se logra identificar la flexibilidad de la musculatura lumbar y de los isquiotibiales (Valencia et al., 2009), que sirve como parámetro para evaluar la flexibilidad a nivel general. Existen medidas específicas que indican el grado de flexibilidad del individuo. Hoeger y Hopkins (1992) exhiben la siguiente valoración para la prueba Sit and Reach modificada en mujeres: baja $<32$; regular 32-36.5; media 36.6-40; buena 40.1-42; excelente 42. Al realizar la prueba pre-test, la sujeto inicialmente obtuvo una medida de $22 \mathrm{~cm}$, la cual aumentó significativamente a $28 \mathrm{~cm}$, en ocho semanas. A pesar de que dichas medidas no se encuentran dentro de los parámetros de valoración normal e indican aún una baja flexibilidad en ella, de acuerdo a Grosser (1992), el haber aumentado la flexibilidad en $6 \mathrm{~cm}$ contribuyó a mejorar su calidad de vida, según lo indica también Lourdes.

De acuerdo con Lourdes, el progreso percibido en la capacidad aeróbica y la flexibilidad mejoraron su calidad de vida, especialmente en su trabajo y en el desarrollo de actividades con familiares, lo cual le permitió compartir más tiempo con estos. 
Los datos determinados en la sujeto de estudio al iniciar la investigación concuerdan con los resultados encontrados por Gurer et al. (2006), donde se encuentra que mujeres con FM mostraron valores de LDL y triglicéridos significativamente más altos que los obtenidos por el grupo control (mujeres sin ninguna patología diagnosticada). También concuerdan con los datos encontrados por Cordero et al. (2012), a partir una investigación en 183 sujetos con FM diagnosticados mediante puntos sensibles y dolorosos; los cuales fueron distribuidos en tres grupos, según el Índice de Masa Corporal (IMC) en: no obesos, con sobrepeso y obesos, así como un análisis del perfil lipídico (PL). Los resultados mostraron correlación entre la presencia de puntos dolorosos y el IMC, además los sujetos con sobrepeso mostraron un mayor incremento en los valores del perfil lipídico.

ACSM (2005) expresa los rangos de referencia para valorar las dislipidemias, situando el valor limítrofe alto de colesterol total (CT) entre $200 \mathrm{mg} / \mathrm{dL}$ y $239 \mathrm{mg} / \mathrm{dL}$, situación en la que se encontraba la paciente antes del tratamiento con actividad física, con un valor de 215 $\mathrm{mg} / \mathrm{dL}$. Este valor disminuyó a $180 \mathrm{mg} / \mathrm{dL}$ posterior al tratamiento, que lo califica como deseable, lo que trae mayores beneficios a la salud, disminuyendo las probabilidades de sufrir complicaciones cardiacas, diabetes e hipertensión, ya que según la ACSM (2005) los niveles alterados tanto en CT como en otras dislipidemias constituyen un factor de riesgo a la salud.

Respecto a las lipoproteínas de baja densidad (LDL), se obtuvo un valor pre-test de $140 \mathrm{mg} / \mathrm{dL}$, situado por encima de $130 \mathrm{mg} / \mathrm{dL}$ el cual está determinado como el valor máximo. Dicho valor también disminuyó a $117 \mathrm{mg} / \mathrm{dL}$ en el post-test, categorizándose al igual que el CT como deseable de acuerdo con la NCEP (2002). Los valores de las proteínas de alta densidad (HDL) fueron de $45 \mathrm{mg} / \mathrm{dL}$, por lo que se clasifica dentro del valor deseado. Aún de esa forma, dicho valor mejoró a $46 \mathrm{mg} / \mathrm{dL}$, de acuerdo con valores establecidos por la NCEP (2002). Finalmente, los triglicéridos en el pre-test fueron de $150 \mathrm{mg} / \mathrm{dL}$, con lo que sitúa en el límite establecido según el NCEP (2002), que lo califica como ideal $<150 \mathrm{mg} / \mathrm{dL}$. Este valor disminuyó a $82 \mathrm{mg} / \mathrm{dL}$ posterior al tratamiento con actividad física. Grundy et al. (2004) recomiendan evitar la inactividad física y la obesidad dado que dichos factores guardan una estrecha relación con los valores de LDL y triglicéridos, lo que incrementa el riesgo cardiovascular a riesgo moderado y riesgo alto, principalmente si estos valores se presentan de forma concomitante con valores anormales en la presión arterial y la frecuencia cardiaca en reposo.

Cabe señalar que posterior a la intervención en esta investigación, el perfil lipídico obtenido por la paciente disminuyó dado que tanto el LDL como los triglicéridos descendieron sus niveles, pasando de valores de riesgo a valores óptimos, y mejoró el HDL, lo que indica que su factor de riesgo también se redujo, con lo cual obtiene no solo beneficios específicos hacia su patología sino también cardiovasculares, lo que de igual forma disminuye factores de riesgo a la salud y preserva su calidad de vida (National Institutes of Health, 2002).

Una vez abordados los aspectos a nivel físico en el presente estudio, se analizaron también aspectos psicológicos alrededor de la fibromialgia. González, Ortín y Bonillo (2011) enfatizan que el tratamiento de la fibromialgia se debe abordar desde diferentes perspectivas y no solo bajo un método, esto debido a que las intervenciones sobre trastornos como la 
ansiedad y la depresión dan mejores resultados cuando se combina la práctica de actividad física y la terapia psicológica. Se destaca por tanto la necesidad de integrar ambos programas en el tratamiento de la FM en busca de una potencialización surgida de proporcionar un enfoque integral.

En este campo se valoraron constructos como la catastrofización del dolor percibido, la autoimagen y el autoconcepto físico. En cuanto al dolor percibido por los pacientes que sufren FM y la catastrofización del mismo, al inicio de la intervención Lourdes obtuvo un puntaje de 40 y finalizada la intervención fue de 17, lo cual es un logro significativo bajar el nivel de dolor en más de la mitad. Según Okifuji, Donaldson, Barck y Fine (2010) mediante un estudio, el $50 \%$ de un total de 215 sujetos con FM tenían obesidad y un $30 \%$ presentaban sobrepeso, asociando la FM a padecimientos de la calidad del sueño y la percepción de dolor. Resultados como este y los obtenidos en el estudio, demuestran que una terapia integral (nutrición-fuerza-capacidad aeróbica y flexibilidad) es un tratamiento efectivo para una persona con fibromialgia, ya que disminuye sus sentimientos pesimistas hacia la vida y brinda mayor esperanza hacia el futuro y con respecto al pronóstico de la enfermedad, así como una mayor calidad de las horas de sueño debido a una menor percepción del dolor.

En la medición de la imagen corporal se puede apreciar que, al inicio de la intervención, al marcar la figura que representaba su estado actual, Lourdes señaló la última imagen (la que indica mayor sobrepeso), mientras terminada la intervención marcó la penúltima figura; es decir, hubo un cambio en la percepción de la imagen de esta persona hacia una mejor autoimagen. En cuanto a la figura que representaba el ideal previo a la intervención, Lourdes señaló la cuarta imagen; mientras que concluido el programa de ejercicio y relajación, marcó la sexta figura. Hubo un cambio en el pensamiento de Lourdes sobre la figura que aspira tener, siendo esta última más realista con su somatotipo y mostrando mayor aceptación de sí misma, lo cual se evidencia tanto a través del test aplicado como por sus comentarios. Estos resultados de percepción de la imagen corporal son similares a lo que indica la literatura, ya que una vez finalizado un programa de ejercicio, las personas tienden a cambiar su percepción de IC a una más positiva (Moreno, Cervelló y Moreno, 2006; Williams y Cash, 2001).

En cuanto a la medición del autoconcepto físico, existieron diferencias positivas en todas las dimensiones (habilidad física, condición física, fuerza, atractivo físico y autoconcepto físico general), excepto en el autoconcepto general, el cual en el pre-test obtuvo 23 puntos, mientras que en el post-test tuvo un punto menos. Estos resultados encontrados coinciden con los de García et al. (2012), quienes aplicaron un programa de ejercicio físico 3 veces a la semana durante 12 semanas a un grupo de mujeres con fibromialgia y encontraron que, además de los beneficios en la aptitud física, el ejercicio físico también mejora la autoestima y el autoconcepto. Los beneficios dados por el ejercicio físico a la autoestima y el autoconcepto se asocian, según García et al (2012), debido a que dichos constructos están estrechamente relacionados con la percepción física del propio cuerpo, por esta razón se da una mejora de la autoimagen. Algo importante de mencionar durante el desarrollo de la intervención es que Lourdes constantemente utilizaba expresiones como: "me siento feliz", "estoy haciendo bien las cosas", "soy guapa", "soy fuerte"; esto indica que es una persona que incrementó su autoestima, lo cual la puede beneficiar mucho en la parte de mantenerse activa físicamente, además de una mayor autoaceptación, favoreciendo su parte social. 
Los resultados de esta investigación concuerda con el obtenido por Rossy et al. (1999), quienes mediante un meta-análisis concluyeron que una intervención óptima para la FM comprende también aspectos no farmacológicos, tales como el ejercicio físico y la terapia cognitivo conductual, que ayuden a mejorar la calidad de vida de esta población. Asimismo, estudios como el realizado por Sarzi et al. (2011) indican que un tratamiento multidisciplinario no farmacológico de la FM puede contribuir a la disminución en la salud mental mediante la disminución en el uso de antidepresivos, opiáceos, sedantes y relajantes musculares, lo que se evidenció en el caso del presente estudio por medio de la entrevista, en la cual la paciente manifestaba mayor capacidad para resolver conflictos personales y familiares, los cuales empeoraban su percepción del dolor físico.

Un estudio similar fue realizado por Sañudo et al. (2010), quienes emplearon ejercicio aeróbico, fuerza muscular y flexibilidad mediante dos sesiones semanales durante 24 semanas. Ellos demostraron que dicha intervención mejoró aspectos de las pacientes tales como mayor vitalidad, mejoras en la función física, la salud mental y la disminución del dolor corporal. Busch, Schachter, Overend y Barner (2008) determinaron, a través de una revisión sistemática, que el ejercicio aeróbico posee efectos beneficiosos para la calidad de vida de las personas con FM y señalan la falta de estudios en la aplicación del entrenamiento contrarresistencia como medio de tratamiento. Sin embargo, Suelen, Leite, Homann, Stefanello y Rodacki (2012) hallaron que las mujeres con FM poseen un déficit en la fuerza muscular en comparación con mujeres sanas, principalmente debido al dolor. En el caso de Lourdes, dicho componente de la actitud física afectaba diariamente sus actividades, tales como cocinar, la limpieza del hogar y en el desempeño de sus labores, sin embargo, posterior a la intervención dichas funciones se vieron mejoradas, probablemente debido al trabajo contrarresistencia.

Dadas las características de la FM, Betina, Rivera, Alegre y Pereda (2006), mediante una revisión sistemática, sugieren que el abordaje ideal para dicha enfermedad debería abarcar diferentes disciplinas del área de la salud. Estos resultados están en concordancia con los obtenidos en la presente investigación, los cuales respaldan que una intervención integral de la FM puede brindar beneficios en el tratamiento de dicha patología. Lo anterior es de suma importancia, dado que la FM se presenta principalmente en la población femenina. Sánchez, Aparicio y Dresch (2006) concluyen, a partir de un estudio, que las variables psicológicas predicen más significativamente la salud física de las mujeres en comparación con los hombres.

En conclusión, el presente estudio sugiere que una intervención de ejercicio físico junto con la consejería nutricional, es una estrategia efectiva para mejorar la calidad de vida y el bienestar de personas que presentan esta patología. Estudios futuros en este ámbito podrían estimar el impacto de un programa integral sobre otras autopercepciones como la autoestima y enfermedades psicológicas como la depresión y la ansiedad. Más allá de un estudio de caso, cabe considerar la aplicación de un programa integral, en una población con fibromialgia, cuya muestra sea amplia. Además, sería importante la terapia psicológica como una forma de complementar la intervención, abarcando todas las áreas posibles del desarrollo humano. 


\section{Referencias Bibliográficas}

American College of Sports Medicine [ACSM] (2005). ACSM's Guidelines for Exercise Testing and Prescription (7 ed.). Philadelphia: Lippincott Williams \& Wilkins.

Akkaya, N., Akkaya, S., Simsir, N., Sengul, C., \& Sahin, F. (2012). Relationship between the body image and level of pain, functional status, severity of depression, and quality of life in patients with fibromyalgia syndrome. Clinical Rheumatology, 31, 983-988. http://dx.doi.org/10.1007/s10067-012-1965-9

Albentosa, A. (2008). Terapia manual como tratamiento de la fibromialgia: caso clínico. Revista Fisioterapia, 7(2), 33-43. Recuperado de http://www.ucam.edu/revistafisio/numeros/volumen-7/numero-2-diciembre-2008/04Terapia\%20manual\%20como\%20tratamiento\%20de\%20la.pdf

Aparicio, V., Ortega, F., Heredia, J., Carbonell, A., \& Delgado, M. (2011). Analysis of the body composition of Spanish women with fibromialgia. Reumatologya Clinica, 7(1), 7-12. Recuperado de http://www.siduna.una.ac.cr:2231/S2173574311700028/1-s2.0S2173574311700028-main.pdf? tid=cafff96e-46a6-11e3-97fd-

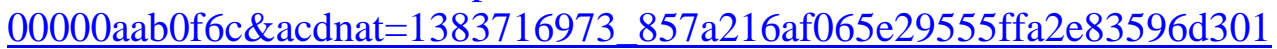

Arcas, M., Gálvez, D., León, J., Paniagua, S. y Pellicer, M. (2004). Manual de Fisioterapia: Fraumatología, afecciones cardiovasculares y otros campos de actuación. España. Editorial Mad, S.L.

Arranz, L., Canela, M. A., \& Rafecas, M. (2012). Relationship between body mass index, fat mass and lean with SF-36 aquality of life scores in a group of fibromyalgia patients. Rheumatology International, 32, 3605-3611. http://dx.doi.org/10.1007/s00296-011$\underline{2250-\mathrm{y}}$

Ayala, F., Saint de Baranda, P., de Ste Croix, M. y Santoja, F. (2012). Fiabilidad y validez de las pruebas sit-and-reach, revisión sistemática. Revista Andaluza de Medicina del Deporte, 05(02), 57-66. http://dx.doi.org/10.1016/S1888-7546(12)70010-2

Betina, M., Rivera, J., Alegre, C. y Pereda, C. (2006). Intervenciones no farmacológicas y tratamientos alternativos en fibromialgia. Medicina Clínica, 127 (8), 295-9. http://dx.doi.org/10.1016/S0025-7753(06)72237-0

Busch, A. J., Schachter, T. J., Overend, P. M., \& Barner, K. (2008). Exercise for fibromyalgia: A systematic review. The Journal of Rheumatology, 35(6), 1130-1144. Recuperado de $\underline{\text { http://scholar.google.com/scholar?cluster }=10057275190543724601 \& h l=e n \& a s \_s d t=0}$, $\underline{5}$

Bragado, C., Hernández, M. J., Sánchez, L. y Urbano, S. (2008). Autoconcepto físico, ansiedad, depresión y autoestima en niños con cáncer y niños sanos sin historia de cáncer. Psicothema, 20(3), 413-419). Recuperado de http://www.unioviedo.es/reunido/index.php/PST/article/view/8675/8539 
Carmona, I. M., Castro, A. M., Matarán, G. A., Gutiérrez, A. B., Ramos, E. y Moreno, C. (2011). Efectos de un programa de ejercicios aeróbicos y técnicas de relajación sobre el estado de ansiedad, calidad del sueño, depresión y calidad de vida en pacientes con fibromialgia, ensayo clínico aleatorizado. Medicina Clínica, 137(9), 398-401. http://dx.doi.org/10.1016/j.medcli.2010.09.045

Castillo, I. y Moncada, J. (2010). El efecto de la frecuencia de participación en un programa de ejercicios contra resistencia sobre la estima y la satisfacción corporal de mujeres universitarias costarricenses. Revista de Iberoamericana de Psicología del ejercicio y del Deporte, 5(2), 195-208. Recuperado de http://www.webs.ulpgc.es/riped/docs/20100202.pdf

Cordero, M., Alcocer, E., Cano, F., Sánchez, B., Fernández, P., Moreno, A., Fernández, A., \& De Miguel, M. (2012). Clinical symptoms in fibromyalgia are associated to overweight and lipid profile. Rheumatology International, 34, 419-422. http://dx.doi.org/10.1007/s00296-012-2647-2

Cotte, V., Faltenbacher, H., Von Willich, W., \& Bogner, R. (2008). Trial of validation of two devices for self-measurement of blood pressure according to the European Society of Hypertension International Protocol: The Citizen CH-432B and the Citizen CH656C.Blood Pressure Monitoring, 13(1), 55-62. Recuperado de http://journals.lww.com/bpmonitoring/Abstract/2008/02000/Trial_of_validation_of_t wo_devices_for.9.aspx

Cox, R. (2008). Psicología del deporte, conceptos y sus aplicaciones (6 ed.). Madrid, España: Editorial Médica Panamericana.

Escudero, M., García, N., Prieto, M., Pérez, O., Carles, J. y López, M. (2010). Fibromialgia: percepción de pacientes sobre su enfermedad y el sistema de salud. Estudio de investigación cualitativa. Reumatología Clínica, 6 (01), 16-22. http://dx.doi.org/10.1016/j.reuma.2009.04.008

Esteve, J., Rivera, J., Vallejo, M., \& Grupo ICAF. (2009). Assesment of functional capacity in fibromyalgia. Comparative analysis of construct validity of three functional scales. Rheumatology Clinical, 6(3), 141-144. Recuperado de http://www.siduna.una.ac.cr:2231/S2173574310700320/1-s2.0-S2173574310700320main.pdf? tid=54557fd4-4762-11e3-8b51-

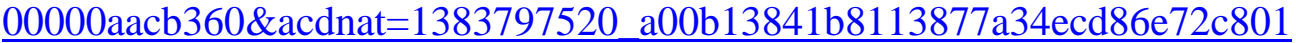

Estrada, N., Munguía, D., Legaz, A. y Serrano, E. (2003). Influencia de un programa de actividad física en la calidad de vida de sujetos diagnosticados de fibromialgia. Universidad de Zaragoza. Recuperado de http://cienciadeporte.eweb.unex.es/congreso/04\%20val/pdf/C124.pdf

Fauci, A., Braunwald, E., Kasper, D., Hauser, S., Longo, D., Jameson, J. y Loscalzo, J. (2008). Harrison principios de medicina interna (17ed). Distrito Federal, México: McGraw-Hill Interamericana Editores. 
Fernández, J., Méndez, A. y Sanz, D. (2007). Fibromialgia y ejercicio físico. Archivos de Medicina del Deporte, 24(118), 121-129. Recuperado de http://femede.es/documentos/Revision_Fibromialgia_121_118.pdf

Garaigordobil, M. y Govillard, L. (2014). Fibromialgia: discapacidad funcional, autoestima y perfil de personalidad. Información Psicológica, 106, 4-16. Recuperado de http://www.informaciopsicologica.info/OJSmottif/index.php/leonardo/article/view/12 $5 / 99$

García, A., De Paz, J., \& Márquez, S. (2012). Effects of an exercise program on self-esteem, self-concept and quality of life in women with fibromyalgia: A randomized controlled trial. Rheumatología Internacional, 32(7), 1869-1876. Recuperado de http://www.siduna.una.ac.cr:2219/content/pdf/10.1007\%2Fs00296-011-1892-0.pdf

García, J., Rodero, B., Alda, M., Sobradiel, N., Montero, J. y Moreno, S. (2008). Validación de la versión española de la escala de la catastrofización ante el dolor (Pain Catastrophizing Scale) en la fibromialgia. Hospital Universitario Miguel Servet, 131(13), 487-492. Recuperado de http://www.elsevier.es/sites/default/files/elsevier/pdf/2/2v131n13a13127277pdf001.pd $\underline{f}$

Gil, C. (2005). Flexitest: Un método innovador de evaluación de la flexibilidad (1ed). Barcelona, España: Paidotribo.

Giraldo, H. (2008). EPOC Diagnóstico y tratamiento integral, con énfasis en rehabilitación pulmonar (3 ed). Bogotá, Colombia: Editorial Médica Internacional LTDA.

González, J., Ortín, F. y Bonillo, J. (2011). Actividad física, asistencia psicológica y niveles de ansiedad y depresión en mujeres con fibromialgia: un estudio descriptivo.

Cuadernos de Psicología del Deporte, 11(1), 59-64. Recuperado de http://www.siduna.una.ac.cr:2068/pdf25 26/pdf/2011/6BOW/01Mar11/59435413.pdf $? \mathrm{~T}=\mathrm{P} \& \mathrm{P}=\mathrm{AN} \& \mathrm{~K}=59435413 \& \mathrm{~S}=\mathrm{R} \& \mathrm{D}=\mathrm{s} 3 \mathrm{~h} \&$ EbscoContent $=\mathrm{dGJyMNLr40Sep6440 \textrm {dv }}$ uOLCmr0uep69Ssa64SbKWxWXS\&ContentCustomer=dGJyMPGvsEuxqbFMuePfge yx44Dt6fIA

Goñi, A., Ruiz de Azua, S. y Liberal, I. (2004). Propiedades psicométricas de un nuevo Cuestionario para la Medida del Autoconcepto Físico. Revista de Psicología del Deporte, 13(2), 195-213. Recuperado de http://ddd.uab.cat/pub/revpsidep/19885636v13n2p195.pdf

Grosser, M. (1992). Principios del Entrenamiento de la Velocidad. Fundamentos, Métodos y Programas. Barcelona, España: Martínez Roca.

Grundy, S., Cleeman, J., Bairey., Luther, L. Hunninghake, D., Pasternak, R., Smith, S., \& Stone, N. (2004). Implications of recent clinical trials for the National Cholesterol Education Program Adult Treatment Panel III Guidelines. Journal of the American College of Cardiology. NCEP Report, 44(3), 720-732. http://dx.doi.org/10.1016/j.jacc.2004.07.001

Revista MHSalud® (ISSN: 1659-097X) Vol. 11. No. 2. Febrero-Agosto, 2015 Artículo protegido por Licencia Creative Commons Attibution-NonComercial-NoDerivs 3.0 Costa Rica 
Gurer, G., Faruk, O., \& Ay, C. (2006). Serum lipid profile in fibromialgia women. Clinical Rheumatology, 25, 300-303. http://dx.doi.org/10.1007/s10067-005-0004-5

Harada, N., Chiu, V., \& Stewart, A. (1999). Mobility-related function in older adults: Assessment with a 6-minute walk test. [abstract]. Archives of Physical Medicine and Rehabilitation, 80(7), 837-841. http://dx.doi.org/10.1016/S0003-9993(99)90236-8

Hoeger, W., \& Hopkins, D. (1992). A comparisson of the sit and reach and the modified sit and reach in the measurement of flexibility in women. Research Quarterly for Exercise and Sport, 63(2), 191-195. http://dx.doi.org/10.1080/02701367.1992.10607580

Martínez, E. (2002). Pruebas de aptitud física (1 ed.). España: Editorial Paidotribo.

Mañex, I., Fenolloza, A., Martínez, A. y Salazar, A. (2005). Calidad del sueño y depresión en fibromialgia. Revista Sociedad Española del Dolor, 12(8), 491-499. Recuperado de http://sid.usal.es/idocs/F8/ART13018/calidad_del_sue\%C3\%B1o.pdf

Moreno, B., Garrosa, E. y Gálvez, M. (2005). Personalidad positiva y salud. Psicología de la salud. Temas Actuales de Investigación en Latinoamérica, 59-76. Recuperado de http://www.uam.es/gruposinv/esalud/Articulos/Psicologia\%20Positiva/PERSONALID AD-POSITIVA-Y-SALUD.pdf

Moreno, J., Moreno, R. y Cervelló, E. (2006). El autoconcepto físico como predictor de la intención de ser físicamente activo. Psicología y Salud, 17, 261-267. Recuperado de http://www.um.es/univefd/PS.pdf

National Institutes of Health [NIH]. (2002). Detection, evaluation, and treatment of high blood cholesterol in adults (Adults treatment Panel III). Third Report of the National Cholesterol Education Program (NCEP) Expert Panel on NIH Publication No. 025215. Recuperado de http://www.nhlbi.nih.gov/guidelines/cholesterol/atp3full.pdf

Okifuji, A., Donaldson, G. W., Barck, L., \& Fine, P. (2010). Relationship between fibromyalgia and obesity in pain, function, mood, and sleep. American Pain Society, 11(12), 1329-1332. http://dx.doi.org/10.1016/j.jpain.2010.03.006

Osman, A., Barrios, B., Gutierrez, P., Kopper, B., Merrifield, T., \& Grittman, L. (2000). The pain catastrofhizing scale: Futher psychometric evaluation with adult. Journal of Behavioral Medice, 23(4), 351-364. http://dx.doi.org/10.1023/A:1005548801037

Pedersen, B., \& Saltin, B. (2006). Evidence for prescribing exercise as therapy in chronic disease. Scandinavian Journal of Medicine \& Science in Sports, 16, 3-63. http://dx.doi.org/10.1111/j.1600-0838.2006.00520.x

Prieto, J. y Yuste, J. (2010). La clínica y el laboratorio. Interpretación de análisis y pruebas funcionales, exploración de los síndromes, cuadrado biológico de las enfermedades. Barcelona, España: Elsevier España, S.L. 
Rodríguez, S. y Cruz, S. (2006). Evaluación de la imagen corporal en mujeres latinoamericanas residentes en Guipúzcoa. Un estudio exploratorio. Anales de Psicología, 22(2), 186-199. Recuperado de http://www.um.es/analesps/v22/v22_2/0322_2.pdf

Rossy, L., Buckelew, S., Dorr, N., Haugglud, K., Thayer, J., McIntosh, M., Hewett, J., \& Johnson, J. (1999). A meta-analysis of fibromyalgia treatment interventions. Annals of Behavioral Medicine, 21(2), 180-191. http://dx.doi.org/10.1007/BF02908299

Sánchez, M. P., Aparicio, M. E. y Dresch, V. (2006). Ansiedad, autoestima y satisfacción autopercibida como predictores de la salud: diferencias entre hombres y mujeres. Psicothema, 18(3), 584-590. Recuperado de http://www.unioviedo.es/reunido/index.php/PST/article/view/8476

Sañudo, B., Galiano, D., Carrasco, L., Blagojevic, M., De Hoyo, M., \& Saxton, J. (2010). Aerobic exercise versus combined exercise therapy in women with fibromyalgia Syndrome: A randomized controlled trial. Archives of Physical Medicine and Rehabilitation, 91(12), 1838-1843. http://dx.doi.org/10.1016/j.apmr.2010.09.006

Sarzi, P., Atzeni, F., Salaffi, F., Cazzola, M., Benucci, M., \& Mease, F. J. (2011). Multidisciplinary approach to fibromyalgia: What is the teaching? Best Practice \& Research Clinical Rheumatology, 25, 311-319. Recuperado de http://ac.elscdn.com.una.idm.oclc.org/S1521694211000301/1-s2.0-S1521694211000301main.pdf? tid=eae45fa6-5431-11e4-9ac8-

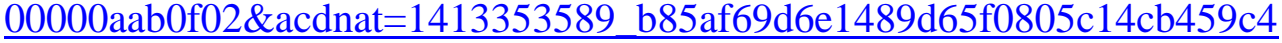

Sebiani, S. (2005). Uso de la Danza-Terapia en la Adaptación Psicológica a Enfermedades Crónicas. Revistas Reflexiones, 84(1), 49-56.Recuperado de http://www.redalyc.org/pdf/729/72920801005.pdf

Sepulveda, A., Btella, J. y León, J. (2001). La alteración de la imagen corporal en los trastornos de la alimentación: un meta análisis. Psicothema, 13, 7-16. Recuperado de http://156.35.33.98/reunido/index.php/PST/article/view/7840/7704

Suelen, M., Leite, N., Shay, B., Homann, D., Stefanello, J., \& Rodacki, A. (2012). Functional capacity, muscle strength and falls in women with fibromyalgia. Clinical

Biomechanics, 27, 578-583. Recuperado de http://ac.elscdn.com.una.idm.oclc.org/S0268003311003226/1-s2.0-S0268003311003226main.pdf? tid=6e2a5a42-567f-11e4-ad5e00000aacb35d\&acdnat $=1413606783$ f133a1 ae268fbd0469be54191ede9108

Sullivan, M., Bishop, S., \& Pivick, J. (1995). The pain catastrophizing scale: Development and validation. Psychological Assessment, 7(4), 524-532. http://dx.doi.org/10.1037/1040-3590.7.4.524

Thompson, M., \& Gray, J. (1995). Development and validation of a new body-image assessment scale. Recuperado de http://www.ncbi.nlm.nih.gov/pubmed/7722852 
Valencia, M., Alonso, B., Alvares, M., Barrientos, M., Ayán, C., \& Martin, V. (2008). Effects of 2 physiotherapy programs on pain reception, muscular flexibility, and illness impact in women with fibromyalgia: A pilot study. Journal of Manipulatuve and Physiological Therapeutics, 32(1), 84-92. Recuperado de http://www.siduna.una.ac.cr:2231/S0161475408002881/1-s2.0-S0161475408002881main.pdf? tid=70e8b988-4765-11e3-991a$\underline{00000 \mathrm{aacb} 35 \mathrm{e} \& \mathrm{acdnat}=1383798856 \_7653087 \mathrm{db} 4539546 \mathrm{dd} 0110 \mathrm{e} 630 \mathrm{fdb} 99 \mathrm{~b}}$

Williams, P., \& Cash, T. (2001). Effects of a circuit weight training program on the body images of college students. International Journal of Eating Disorders, 30(1), 75-82. http://dx.doi.org/10.1002/eat.1056

Recepción: 20 de noviembre del 2014.

Corrección: 12 de enero del 2015.

Aceptación: 14 de enero del 2015.

Publicación: 31 de enero del 2015. 